Article

\title{
Simultaneous Sensing of Temperature and Bi-Directional Strain in a Prismatic Li-Ion Battery
}

\author{
Micael Nascimento* (1), Marta S. Ferreira ${ }^{(1)}$ and João L. Pinto \\ Department of Physics and I3N, University of Aveiro, Campus de Santiago, 3810-193 Aveiro, Portugal; \\ marta.ferreira@ua.pt (M.S.F.); jlp@ua.pt (J.L.P.) \\ * Correspondence: micaelnascimento@ua.pt; Tel.: +351-234-370-356; Fax: +351-234-378-197
}

Received: 17 April 2018; Accepted: 4 May 2018; Published: 10 May 2018

check for updates

\begin{abstract}
Thermal and pressure stability of Li-ion batteries $(\mathrm{LiB})$ are the most important parameters for safety. In abuse operating conditions, the rapid increase of temperature and pressure can cause the appearance of hot-spots, which may lead to an increasing degradation rate or even to the battery's explosion and/or combustion. A sensing network of fiber Bragg gratings is attached to the surface of a prismatic $\mathrm{LiB}$ to monitor its temperature and bi-directional strain variations through normal charge $(0.70 \mathrm{C})$ and two different discharge rates $(1.32 \mathrm{C}$ and $5.77 \mathrm{C})$ in the $x$ - and $y$-directions. More significant variations are registered when the $\mathrm{LiB}$ operates in abnormal conditions. A maximum temperature variation of $27.52 \pm 0.13{ }^{\circ} \mathrm{C}$ is detected by the sensors located close to the positive electrode side. Regarding strain and consequent length variations, maximum values of $593.58 \pm 0.01 \mu \varepsilon$ and $51.05 \pm 0.05 \mu \mathrm{m}$ are respectively obtained by the sensors placed on the $y$-direction. The sensing network presented can be a solution for the real-time monitoring, multipoint and in operando temperature and bi-directional strain variations in the LiBs, promoting their safety.
\end{abstract}

Keywords: Li-ion batteries; fiber Bragg grating sensor; strain; temperature; safety

\section{Introduction}

Lithium-ion batteries ( $\mathrm{LiB})$ are extensively used as power sources for a wide range of electronic devices such as smartphones and laptops, ensuring optimized conditions in the perspective of power, energy, long cycle-life, and slow self-discharge [1,2]. Prismatic LiBs, characteristically used on smartphones, are more disposed to thermal and mechanical abuses from external actions.

The induced strain can be a question that affects the LiB stability and safety, making it the principal cause of material cracking and other forms of performance degradation [3]. Analogous to other electrochemical energy storage systems, the chemical compositions of the active materials change under the charge/discharge processes, which induces strains in electrode particles and causes changes in LiB volume. The aim of thickness reduction of smartphones can be a problem for the users, because these new designs of the smartphone do not integrate a dually protective device which relieves at a set pressure, thus avoiding the overpressure of the LiB. Additionally, LiB companies pursue higher energy density and thinner devices at the cost of safety, which moves against the inherently safer design of a commercial LiB [4].

In addition to strain, thermal runaway is also an essential issue, with impact in the global LiB performance, which is reproduced by the fast increase of temperature. The internal structures of LiBs are made-up of multiple layers, forming a 'jelly roll' structure, where each layer consists of the anode, cathode, electrolyte, and polymer film separators. Under abnormal conditions, such as temperature exceeding the separators melting point, mechanical deformation, or breakdown of the layered materials, an internal short circuit can happen. The hot spot generated by internal short circuit can ignite thermal runaway, leading to fire or explosion of the LiB $[5,6]$. 
The ability to quantify and evaluate the mechanism of strain and thermal runaway generated during the electrochemical processes that the batteries can operate will be beneficial information regarding their behavior as well as an active tool to promote their safety. The temperature and strain sensing of LiB is typically performed using thermocouples [7,8], pyrometers [9], electro-mechanical sensors [10], and 3D digital image correlation [6]. Due to their ease in multiplexing, fast response, immunity to electromagnetic interference advantages when compared to electronic sensors [11], recent works showed that fiber Bragg gratings (FBG) are an effective method to perform temperature and strain measurements in LiBs [12-16].

In this work, through a sensing network of FBGs, quantitative temperature, bi-directional strain shifts, and correspondent longitudinal and transversal variations are provided, as a function of the respective voltage signal of a prismatic LiB, during different cycling protocols.

\section{Materials and Methods}

\subsection{FBG Sensors: Mechanism of Operation}

Typically, a FBG sensor consists of a short segment of a single-mode optical fiber (with a length of a few millimeters) with a photoinduced periodically modulated index of refraction in the core of the fiber. The FBG resonant wavelength is related to the effective refractive index of the core mode $\left(n_{\text {eff }}\right)$ and to the grating period $(\Lambda)$. When the grating is illuminated with a broadband optical source, the reflected power spectrum presents a sharp peak (with a full width at half maximum of a few nanometers), which is caused by interference of light with the planes of the grating and can be described through Equation (1) [11]

$$
\lambda_{B}=2 n_{e f f} \Lambda,
$$

where $\lambda_{B}$ is the so-called Bragg wavelength. When the fiber is exposed to external variations of a given measurand (such as strain, temperature, stress or pressure, among others), both $n_{\text {eff }}$ and $\Lambda$ can be altered, causing a shift in the Bragg wavelength.

The FBG sensitivity towards a given parameter is obtained simply by subjecting the sensor to pre-determined and controlled variations of such parameter and measuring the Bragg wavelength for each step. In the case of a linear response, the sensitivity $(K)$ is given by the slope of the linear fit obtained from the experimental data. The effects of temperature are accounted for in the Bragg wavelength shift by differentiating Equation (1)

$$
\Delta \lambda=2 \lambda_{B}\left(\frac{1}{n_{e f f}} \frac{\partial n_{e f f}}{\partial T}+\frac{1}{\Lambda} \frac{\partial \Lambda}{\partial T}\right) \Delta T=\lambda_{B}(\alpha+\xi) \Delta T=K_{T} \Delta T
$$

where $\alpha$ and $\xi$ are the thermal expansion and thermo-optic coefficient of the optical fiber material, respectively. On the other hand, if the fiber is subjected to strain variations, its response can be determined by differentiating Equation (1)

$$
\Delta \lambda=\lambda_{B}\left(\frac{1}{n_{e f f}} \frac{\partial n_{e f f}}{\partial \varepsilon}+\frac{1}{\Lambda} \frac{\partial \Lambda}{\partial \varepsilon}\right) \Delta \varepsilon=\lambda_{B}\left(1-p_{e}\right) \Delta \varepsilon=K_{\varepsilon} \Delta \varepsilon,
$$

where $p_{e}$ is the photoelastic constant of the fiber $(\sim 0.22)$ and $\Delta \varepsilon$ is the applied strain. The strain variations can be determined using the equation $\Delta \varepsilon=\Delta L / L$ where $\Delta L$ is the length variation and $L$ the fiber length over which strain is applied.

\subsection{Strain and Temperature Discrimination: Reference FBG Method}

The temperature and bi-directional strain discrimination were performed using the reference FBG method. On a single measurement of the Bragg wavelength-shift, it is not possible to discriminate the effect of changes in strain and temperature. Therefore, a reference is required for temperature 
measurement. Several methods have been addressed in the literature for an accurate discrimination between these two parameters [17-19]. The most straightforward way is to use a separated, strain-free FBG, which acts as a temperature sensor. In this case, the wavelength shift, $\Delta \lambda_{B 1}$, is related to temperature variations, $\Delta T_{1}$, according to

$$
\Delta \lambda_{B 1}=K_{T 1} \Delta T_{1}
$$

where $K_{T 1}$ is the temperature sensitivity of the non-fixed FBG. This reference FBG is in the same thermal environment as the other sensors, which simultaneously detect strain and temperature. Assuming that the wavelength-shifts to strain and temperature are linear, a response to a strain change, $\Delta \varepsilon_{2}$ and a temperature change, $\Delta T_{2}$, is given by

$$
\Delta \lambda_{B 2}=K_{\varepsilon 2} \Delta \varepsilon_{2}+K_{T 2} \Delta T_{2}
$$

where $K_{\varepsilon 2}$ and $K_{T 2}$ are the strain and temperature sensitivities of the fixed FBG [11], determined in the calibration procedure. Therefore, using Equation (4), the wavelength variations detected by the strain-free FBG can be converted into temperature variations. These temperature variations are then considered in Equation (5), allowing the determination of strain.

\subsection{Experimental Setup}

A network of five FBG sensors, recorded in two different fibers, was placed in direct contact with the LiB surface on the $x$ - and $y$-directions, as schematized in Figure 1 . The FBGs network used in the monitoring system, with estimated gratings length of $\sim 3.0 \mathrm{~mm}$, was pre-fabricated in our lab and written in commercial photosensitive single mode fiber (125 $\mu \mathrm{m}$ of cladding diameter) (FiberCore PS1250/1500) by the phase mask method. The UV radiation system used was a pulsed excimer laser (KrF) (Industrial Bragg Star, Coherent, CA, USA), emitting at a wavelength of $248 \mathrm{~nm}$, $4 \mathrm{~mJ} /$ pulse (20 ns duration), and $500 \mathrm{~Hz}$ repetition rate.

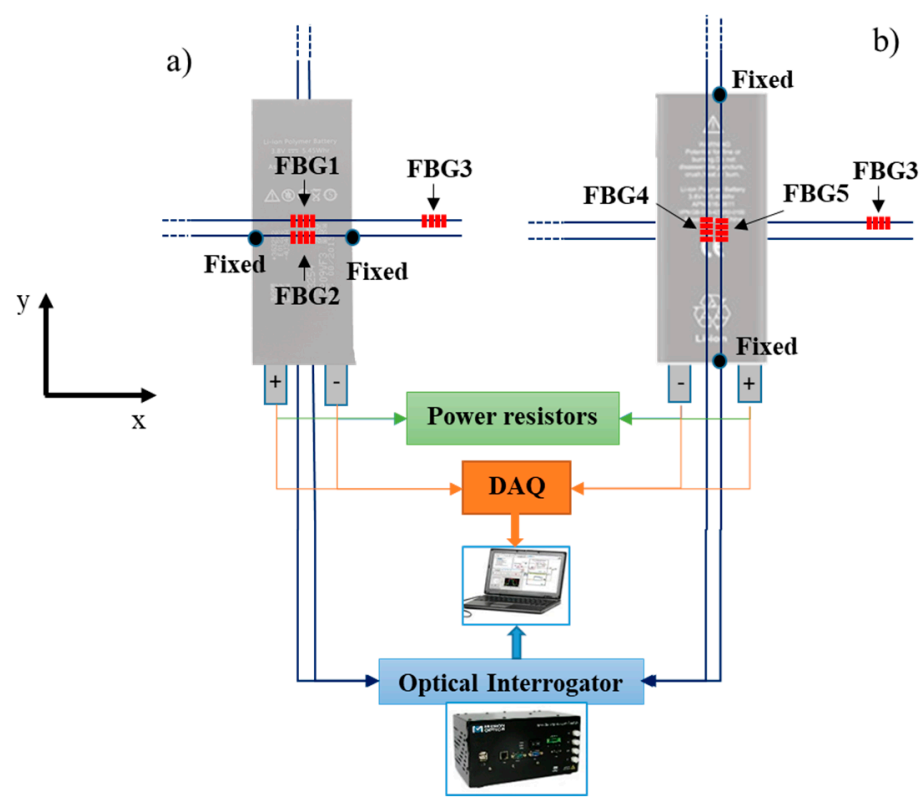

Figure 1. Experimental setup diagram of the temperature and bi-directional strain monitoring system of the battery: (a) down view; (b) top view.

As can be seen in Figure 1, the FBG1 was placed on the $x$-direction, FBG4 on the $y$-direction and FBG3 out of the battery to monitor the external room temperature. These sensors were only used to 
measure temperature variations, as they were not fixed to the battery. The simultaneous detection of the strain and temperature variations was accomplished by fixing a second fiber side by side to the first one, with two sensors: FBG2 and FBG5. This fiber was pre-tensioned and fixed to the battery on its extremities, along the $\operatorname{LiB} x$ - and $y$-directions. Thermal paste was placed along both fibers to increase thermal conductivity between the battery and the FBGs. To calculate the length variations on the two directions, the distance between the two fixed points was considered. As the glue was placed as close to the edge of the $\mathrm{LiB}$ as possible, the length variations are related to the battery dimension.

The $\mathrm{LiB}$ used in this work was a commercial hard prismatic rechargeable smartphone $\mathrm{LiB}$ (Iphone $5 \mathrm{G}$ Battery, Singapore) with an open-circuit voltage of $4.30 \mathrm{~V}$, nominal capacity of $1440 \mathrm{mAh}$, cut-off voltage of $3.20 \mathrm{~V}$, dimensions of 8.6 (length) $\times 3.0$ (width) $\times 0.3 \mathrm{~cm}$ (thickness), and mass of $23.47 \mathrm{~g}$. Inside the aluminum-plastic pouch, the internal structure is constructed by winding the ribbon-like electrode and separator to form a 'jelly-roll' structure. The negative and positive active materials are coated on each side of the separator materials, and thin layers of copper and aluminum foils are used as the current collectors [4].

Prior to the fiber sensor network's attachment to the $\mathrm{LiB}$, a calibration to strain and temperature was carried out. The strain characterization was performed using a micrometric translation stage between $0 \mu \varepsilon$ and $2000 \mu \varepsilon$. The sensors thermal calibration was made on a thermal chamber (Model 340, Challenge Angelantoni Industrie, Massa Martana, Italy). The temperature range was between $20.0^{\circ} \mathrm{C}$ and $50.0^{\circ} \mathrm{C}$, in steps of $5.0^{\circ} \mathrm{C}$. The temperature and strain sensitivities obtained are presented in Table 1.

Table 1. Temperature and strain sensitivities of the FBG sensors

\begin{tabular}{ccccc}
\hline FBG & Direction & $\lambda_{B} / \mathbf{n m}$ & $\boldsymbol{k}_{\boldsymbol{T}} \pm \mathbf{0 . 1 3} / \mathbf{p m} /{ }^{\circ} \mathbf{C}$ & $\boldsymbol{k}_{\varepsilon} \pm \mathbf{0 . 0 1} / \mathbf{p m} / \boldsymbol{\mu \varepsilon}$ \\
\hline 1 & $x$ & 1553.57 & 7.92 & - \\
2 & $x$ & 1553.32 & 7.92 & 1.16 \\
3 & Room temperature sensor & 1540.17 & 8.04 & - \\
4 & $y$ & 1535.70 & 7.80 & - \\
5 & $y$ & 1535.42 & 7.80 & 1.20 \\
\hline
\end{tabular}

The reflected Bragg wavelengths were measured with an optical interrogator (sm125-500, Micron Optics Inc., Atlanta, GA USA) operating at $2.0 \mathrm{~Hz}$ and wavelength accuracy of $1.0 \mathrm{pm}$. The voltage signal was monitored using a 12-bit resolution data acquisition system (DAQ) (USB6008, National Instruments, Austin, TX, USA). The acquisition modules were controlled by a LabVIEW ${ }^{\circledR}$ customized application, allowing the real-time monitoring of the acquired data. The LiB was cycled twice under two different discharge rates of $1.32 \mathrm{C}$ and $5.77 \mathrm{C}$ (where $1.00 \mathrm{C}$ is the rate of the ideal capacity charge/discharge in $1 \mathrm{~h}$ ) through two power resistors of $2.0 \Omega$ and $0.47 \Omega$ until a voltage of $1.95 \mathrm{~V}$ was reached. A normal charge rate of $0.70 \mathrm{C}$ was applied, using a commercial battery charger with output voltage of $5.00 \mathrm{~V}$ (ETA0U83EWE, Samsung, Vietnam).

\section{Results and Discussion}

The temperature variations registered by the FBG sensors placed on the two sides of the battery are presented in Figures 2 and 3. The positive and negative electrodes indicated in the Figures correspond to the sensors placed on the $y$ - and $x$-directions, respectively. The cycling protocol, where the discharge rate of $1.32 \mathrm{C}$ was applied, is shown in Figure 2. Over the CC charge step (1), a maximum temperature variation of $1.29 \pm 0.13{ }^{\circ} \mathrm{C}$ was measured by the sensors placed on the positive electrode side, whereas on the negative electrode side, the maximum temperature shift was of $0.86 \pm 0.13^{\circ} \mathrm{C}$. Between the constant current (CC) charge/discharge steps, constant voltage (CV) steps (2) of $10 \mathrm{~min}$ and $15 \mathrm{~min}$ were selected to stabilize the temperature and relax the battery. 


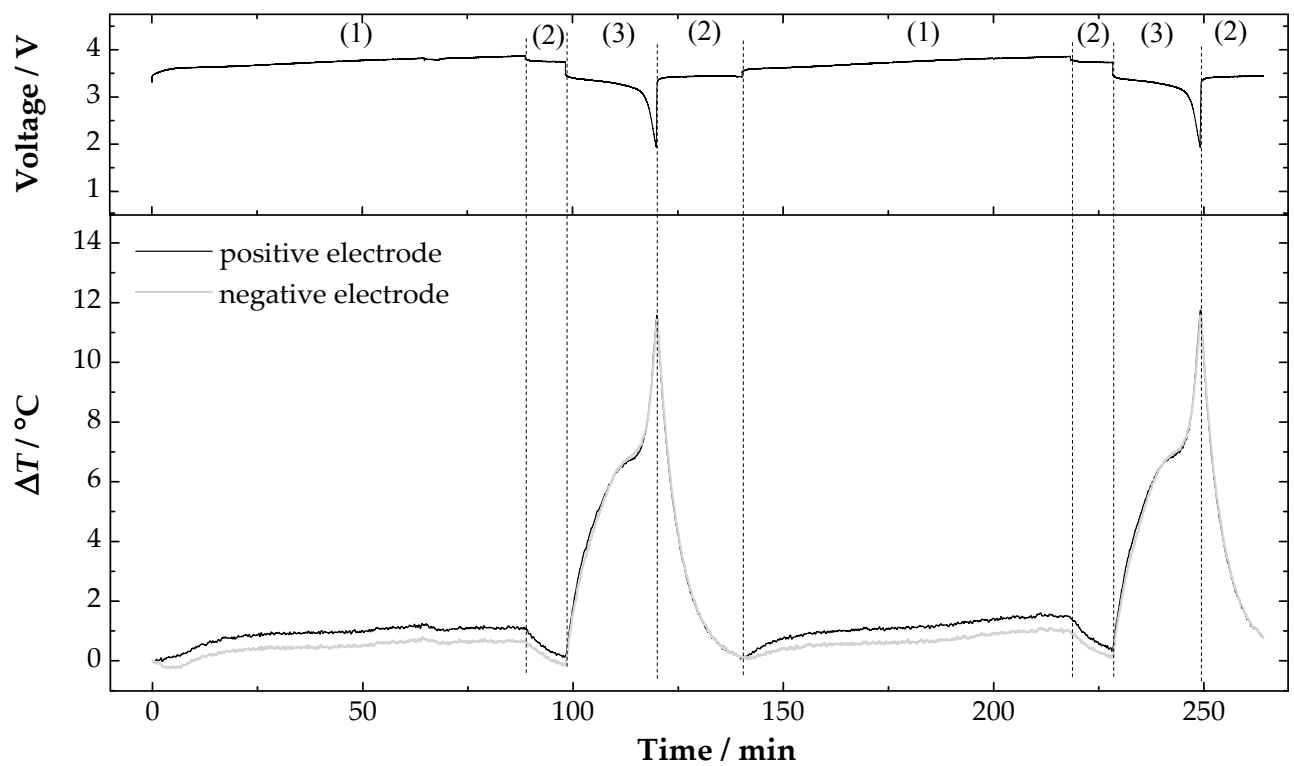

Figure 2. Voltage and temperature variations recorded during two cycling tests, in the sides of the positive and negative electrodes of the battery. (1) CC charge; (2) CV; (3) CC discharge at $1.32 \mathrm{C}$.

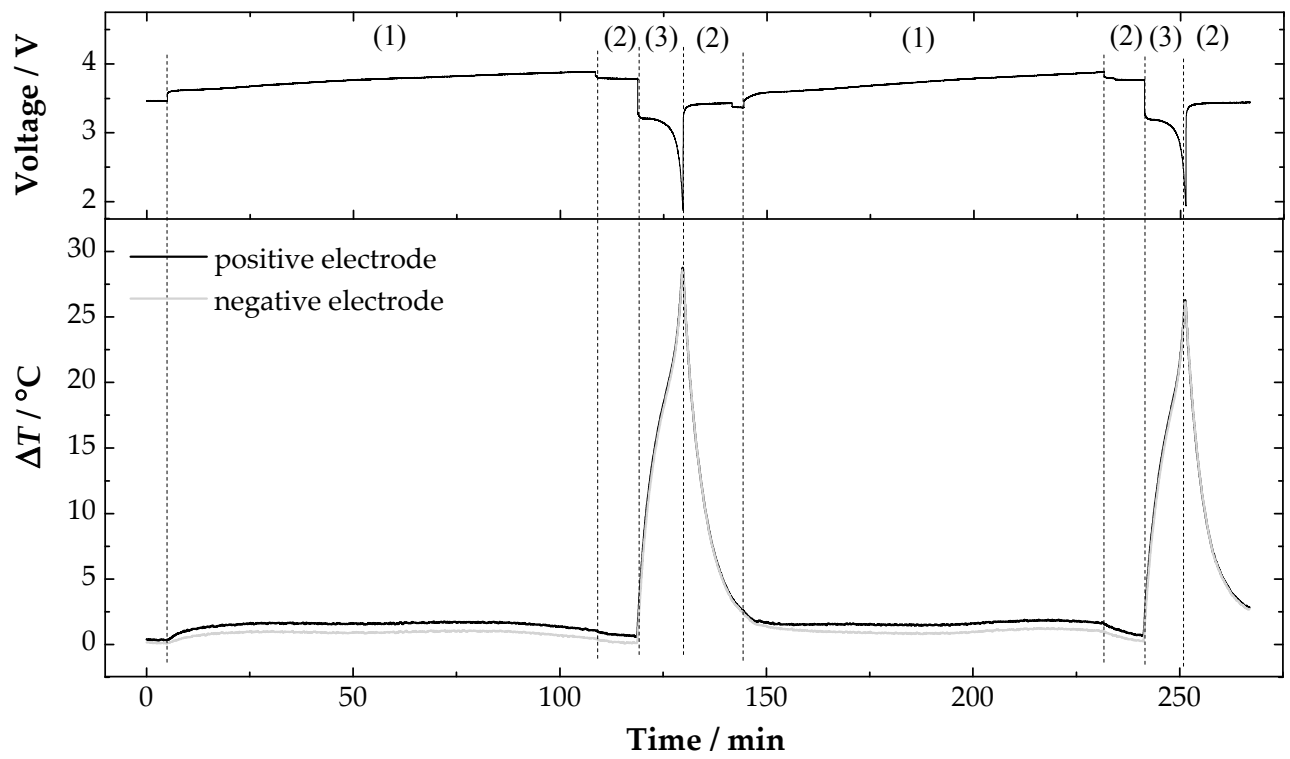

Figure 3. Voltage and temperature variations recorded during two cycling tests, in the sides of the positive and negative electrodes of the battery. (1) CC charge; (2) CV; (3) CC discharge at $5.77 \mathrm{C}$.

The starting of the CC discharge step (3) causes an instantaneous increase on the battery surface temperature, however, the maximum temperature shift was achieved only at the end of the discharge process. These fast and significant increases are related to the larger electrochemical reactions produced by the flow of $\mathrm{Li}+$ ions on migration to the positive electrode. During all the discharge steps, two different moments of temperature variations can also be observed. The first moment occurs when the LiB voltage crosses the cut-off voltage, and the second between the cut-off and the end of the discharge process. The maximum temperature variations detected were very similar on both electrodes, around $11.50 \pm 0.13^{\circ} \mathrm{C}$.

Figure 3 shows the cycling protocol where a discharge rate of $5.77 \mathrm{C}$ was applied. Attending to the CC charge steps (1) and comparing with previous cycling protocol, the same range of values was 
registered by the FBG sensors located on the positive and negative electrodes. As expected, the main difference was in the temperature variations achieved during the CC discharge step (3). In all discharge steps, a monotonic increase can be observed, until the LiB reaches $1.95 \mathrm{~V}$. A maximum temperature variation of $27.52 \pm 0.13{ }^{\circ} \mathrm{C}$ was recorded on the positive electrode side by the FBG4. On the negative electrode side, an inferior $\Delta T$ value of $27.25 \pm 0.13{ }^{\circ} \mathrm{C}$ was obtained, however, this difference is not significant, taking the measurement error into account.

The bi-directional strain variations detected by the FBG sensors on the $x$ - and $y$-directions, during the experimental cycling protocol with the discharge rate of $1.32 \mathrm{C}$, as well as their voltage signal, are presented in Figure 4. Over the CC charge steps (1), mean strain variations of $122.49 \pm 0.01 \mu \varepsilon$ and $29.61 \pm 0.01 \mu \varepsilon$ were measured on the $y$ - and $x$-directions, respectively, when the battery reached the $3.95 \mathrm{~V}$. As already mentioned, during the $10 \mathrm{~min}$ CV step (2), between the CC charge (1) and CC discharge (3) steps, a relaxation occurs on the battery. This relaxation was higher on the $y$-direction, translating into a strain diminishing of $25.58 \pm 0.01 \mu \varepsilon$.

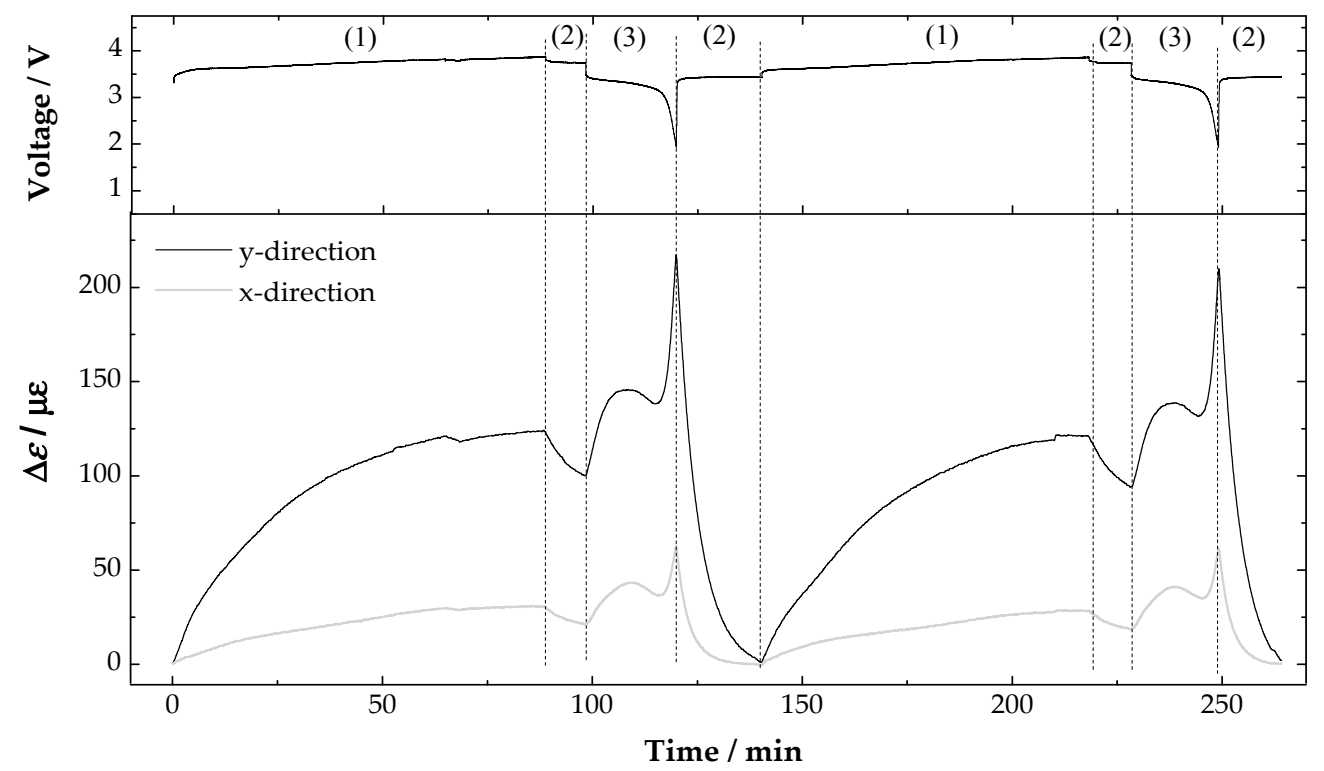

Figure 4. Voltage and strain variations recorded during two cycling tests in the battery in the $x$ - and y-directions. (1) CC charge; (2) CV; (3) CC discharge at $1.32 \mathrm{C}$.

When the battery is subjected to the CC discharge step (3), at $1.32 \mathrm{C}$, three different behaviors can be observed on the two different directions. In the first one, until the LiB reached the $3.30 \mathrm{~V}$, there is a sudden increase of strain. In the $y$-direction this increase is nearly two-times higher than the one measured in the $x$-direction. After this, a strain decreases of $7.39 \pm 0.01 \mu \varepsilon$ and $6.46 \pm 0.01 \mu \varepsilon$ was measured in the $y$ - and $x$-directions, respectively, until the cut-off voltage was reached. This was followed by a fast increase of strain, as the battery starts to operate under abusive conditions, between $3.20 \mathrm{~V}$ and $1.95 \mathrm{~V}$. In this case, the $y$-direction strain increased up to $213.53 \pm 0.01 \mu \varepsilon$. On the $x$-direction, a 3.4-times lower strain value was recorded.

These values can be converted to length variations $(\Delta L)$, by multiplying the strain by the length or the width of the LiB on the $y$ - or $x$-directions, respectively. Thus, maximum $\Delta L$ of $18.36 \pm 0.05 \mu \mathrm{m}$ and $1.86 \pm 0.05 \mu \mathrm{m}$ on the $y$ - and $x$-directions were calculated, respectively. These length variations can be translated to a longitudinal and transversal variation in the battery of $0.021 \%$ and $0.006 \%$ in the $y$ - and $x$-directions, respectively.

The same LiB was submitted to a different cycling protocol test, where a different discharge rate of $5.77 \mathrm{C}$ was applied. This higher discharge rate was selected so that the battery would operate 
under abnormal conditions. However, the same charge rate was used to charge the battery at 3.95 V. The bi-directional strain variations obtained are presented in Figure 5.

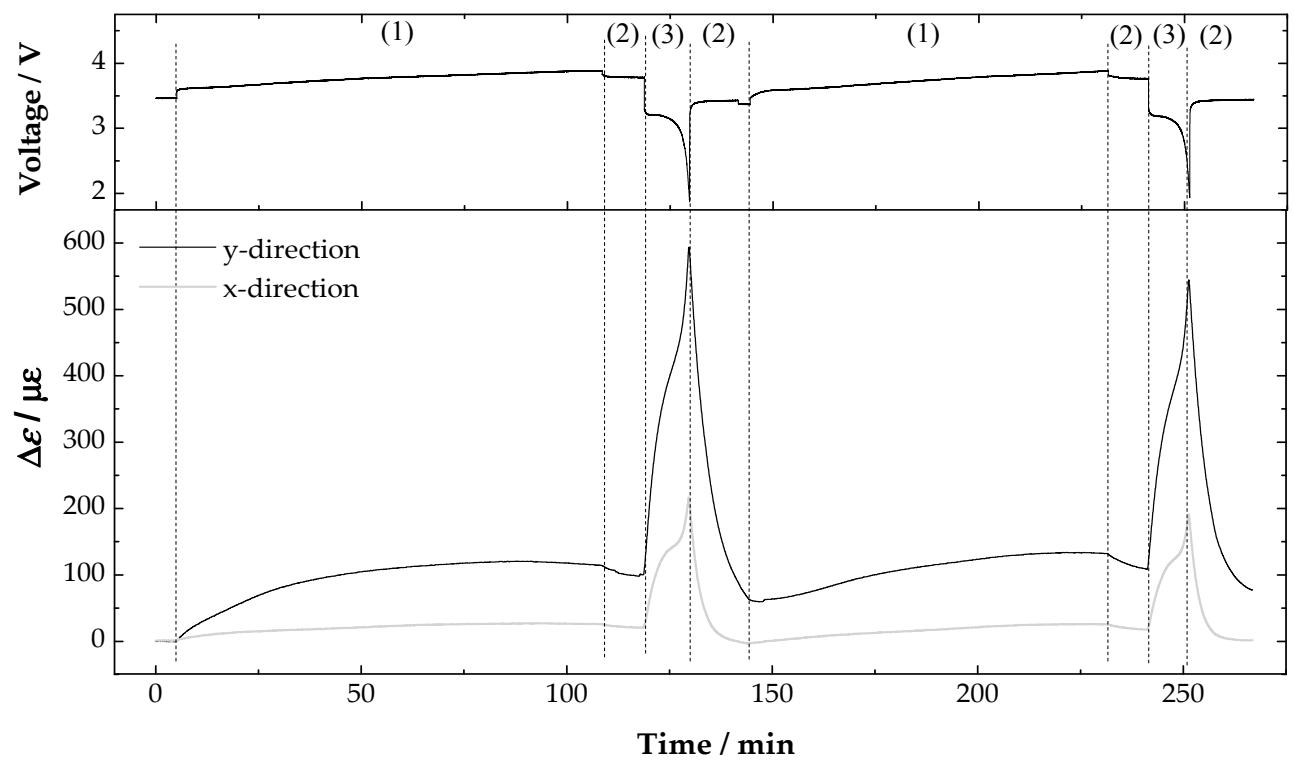

Figure 5. Voltage and strain variations recorded during two cycling tests in the battery in the $x$ - and y-directions. (1) CC charge; (2) CV; (3) CC discharge at $5.77 \mathrm{C}$.

Comparatively to the cycling protocol presented previously and attending the CC charge steps (1) and CV steps (2), the same range of $\Delta \varepsilon$ were obtained, causing maximum $\Delta L$ of $10.53 \pm 0.05 \mu \mathrm{m}$ and $0.89 \pm 0.05 \mu \mathrm{m}$ in the $y$ - and $x$-directions, respectively, and longitudinal and transversal shifts of $0.012 \%$ and $0.003 \%$, respectively, on the battery.

When the battery is subjected to CC discharge steps (3), a monotonic increase can be observed in both directions. However, a different increasing time occurs, before and after the battery reached the cut-off voltage, following the fast voltage decrease. On the $y$-direction, a maximum strain variation of $593.58 \pm 0.01 \mu \varepsilon$ was measured and a correspondent $\Delta L$ of $51.05 \pm 0.05 \mu \mathrm{m}$ was calculated. This high elongation translated in a battery longitudinal variation of $0.060 \%$. The sensors located in the $x$-direction followed a similar behavior as in the previous cycling protocol and detected lower strain variations. In this case, a maximum transversal variation of $0.022 \%$ was obtained.

In all cycles, it was observed that the maximum strain variations are registered when the battery discharge process ends. A relation between the different discharge rates which are applied and the longitudinal and transversal variations on the LiB can be performed by considering the battery dimensions and the $\Delta L$ values obtained in the two directions. For instance, the ratio of the LiB dimensions is 2.87 , whereas dividing the longitudinal by the transversal variation measured over the higher discharge rate, a ratio of 2.73 is calculated. Making the same analogy but for the discharge rate of $1.32 \mathrm{C}$, a ratio value of 3.50 is obtained. This means that the expansion and contraction of the internal 'jelly roll' structure that constitutes the prismatic battery behaves differently on each charge/discharge process which is subject, and it is not proportional to the $\mathrm{LiB}$ dimensions.

Through a complete analysis of the cycling test, it is evident that, in both cases, the maximum values obtained for the temperature and strain variations agree with the sudden decrease of the voltage. Considering all strain and temperature values measured, different behaviors in terms of direction of expansion and temperature variations on the two sides of the battery were detected. These results are in good agreement with the ones found in the literature $[6,9,10]$.

The monitoring of the internal and external parameters during charge and discharge cycles, combined with battery management systems, seems to be adequate to improve overall safety. They also 
could be used to determine realistic operating conditions and geometries, towards more stable cell pack designs.

\section{Conclusions}

A network of FBG sensors was successfully used to simultaneously monitor temperature and bi-directional ( $x$ - and $y$-direction) strain in a prismatic rechargeable $\mathrm{LiB}$, under an experimental cycling protocol with normal CC charge and different CC discharge steps (1.32 C and $5.77 \mathrm{C})$. When the battery was subjected to abnormal operating conditions, as fast discharge and operating below the cut-off voltage, it is evident that higher temperature and strain variations occur, which are promoted by the rapid $\mathrm{Li}+$ transport between the positive and negative electrodes. Over the CC charge step, maximum temperature and strain variations were reached at the end of the process, with values of $1.29 \pm 0.13{ }^{\circ} \mathrm{C}$ in the positive electrode side and $122.49 \pm 0.01 \mu \varepsilon$ and $29.61 \pm 0.01 \mu \varepsilon$ on the $y$ - and $x$-directions, respectively.

During the CC discharge step, the higher strain and temperature values of $593.58 \pm 0.01 \mu \varepsilon$ and $27.52 \pm 0.13{ }^{\circ} \mathrm{C}$ were respectively register for the higher discharge rate and when the LiB voltage was lower. These values also relate to the $y$-direction and positive electrode side and correspond to a battery longitudinal expansion of $0.060 \%$. As expected, there is a deformation increase when the temperature also increases, due the thermal expansion of the materials that compose the battery. Thus, the internal structure of the battery is an important parameter to have in consideration and can influence the behavior of battery materials in terms of expansion and contraction over its operation.

The sensing network presented proved to be an effective, precise, alternative solution to real time monitor, multipoint and in operando temperature and bi-directional strain changes in the LiBs promoting their safety.

Author Contributions: All authors contributed equally. Micael Nascimento and Marta S. Ferreira conceived the experiments and analyzed the data. All authors wrote and revised the paper.

Acknowledgments: This work was funded by FEDER funds through the COMPETE 2020 Program and National Funds through FCT2014 Portuguese Foundation for Science and Technology under the project UID/CTM/50025/2013. Micael Nascimento and Marta S. Ferreira are also grateful for research fellowships BI/UI96/6642/2016 and SFRH/BPD/124549/2016, respectively.

Conflicts of Interest: The author declares no conflicts of interest.

\section{References}

1. Zhang, Z.; Ramadass, P.; Fang, W. Safety of Lithium-ion Batteries. In Lithium-Ion Batteries: Advances and Applications, 1st ed.; Elsevier: Amsterdam, The Netherlands, 2014; Volume 18, pp. 409-435; ISBN 978-0-444-59513-3.

2. Spotnitz, R.; Franklin, J. Abuse behavior of high-power, lithium-ion cells. J. Power Sources 2003, 113, 81-100. [CrossRef]

3. Mankowski, P.J.; Kanevski, J.; Bakirtzian, P.; Cugno, S. Cellular phone collateral damage: A review of burns associated with lithium battery powered mobile devices. Burns 2016, 42, e61-e64. [CrossRef] [PubMed]

4. Duh, Y.S.; Lin, K.H.; Kao, C.S. Experimental investigation and visualization on thermal runaway of hard prismatic lithium-ion batteries used in smart phones. J. Therm. Anal. Calorim. 2018, 1-16. [CrossRef]

5. Leung, P.K.; Moreno, C.; Masters, I.; Hazra, S.; Conde, B.; Mohamed, M.R.; Dashwood, R.J.; Bhagat, R. Real-time displacement and strain mappings of lithium-ion batteries using three-dimensional digital image correlation. J. Power Sources 2014, 271, 82-86. [CrossRef]

6. Wang, Q.; Ping, P.; Zhao, X.; Chu, G.; Sun, J.; Chen, C. Thermal runaway caused fire and explosion of lithium ion battery. J. Power Sources 2012, 208, 210-224. [CrossRef]

7. Mutyala, M.S.K.; Zhao, J.; Li, J.; Pan, H.; Yuan, C.; Li, X. In situ temperature measurement in lithium-ion battery by flexible thin film thermocouples. J. Power Sources 2014, 260, 43-49. [CrossRef]

8. Fu, Y.; Lu, S.; Li, K.; Liu, C.; Cheng, X.; Zhang, H. An experimental study on burning behaviors of 18650 lithium ion batteries using a cone calorimeter. J. Power Sources 2015, 273, 216-222. [CrossRef] 
9. Panchal, S.; Dincer, I.; Agelin-Chaab, M.; Fraser, R.; Fowler, M. Experimental temperature distributions in a prismatic lithium-ion battery at varying conditions. Int. Commun. Heat Mass Transf. 2016, 71, 35-43. [CrossRef]

10. Wang, X.; Sone, Y.; Segami, G.; Naito, H.; Yamada, C.; Kibe, K. Understanding volume change in Lithium-ion cells during charging and discharging using in situ measurements. J. Electrochem. Soc. 2007, 154, A14-A21. [CrossRef]

11. Grattan, K.T.V.; Meggitt, B.T. Optical Fiber Sensor Technology: Applications and Systems; Kluwer Academic: London, UK, 1999; Volume 3, pp. 357-363. ISBN 978-1-4757-6077-4.

12. Yang, G.; Leitão, C.; Lib, Y.; Pinto, J.L.; Jiang, X. Real-time temperature measurement with fiber Bragg sensors in lithium batteries for safety usage. Measurement 2013, 46, 3166-3172. [CrossRef]

13. Nascimento, M.; Novais, S.; Leitão, C.; Domingues, M.F.; Alberto, A.; Antunes, P.; Pinto, J.L. Lithium Batteries Temperature and Strain Fiber Monitoring. In Proceedings of the 24th International Conference on Optical Fiber Sensors, Curitiba, Brazil, 28 September-2 October 2015; Volume 9634 96347V-1. [CrossRef]

14. Sommer, L.W.; Kiesel, P.; Ganguli, A.; Lochbaum, A.; Saha, B.; Schwartz, J.; Bae, C.-J.; Alamgir, M. Fast and slow ion diffusion processes in lithium-ion pouch cells during cycling observed with fiber optic strain sensors. J. Power Sources 2015, 296, 46-52. [CrossRef]

15. Novais, S.; Nascimento, M.; Grande, L.; Domingues, M.F.; Antunes, P.; Alberto, A.; Leitão, C.; Oliveira, R.; Koch, S.; Kim, G.T.; et al. Internal and external temperature monitoring of a Li-ion battery with fiber Bragg grating sensors. Sensors 2016, 16, 1394. [CrossRef] [PubMed]

16. Nascimento, M.; Ferreira, M.; Pinto, J.L. Real time thermal monitoring of lithium batteries with fiber sensors and thermocouples: A comparative study. Measurement 2017, 111, 260-263. [CrossRef]

17. Jin, W. Simultaneous measurement of strain and temperature: Error analysis. Opt. Eng. 1997, 36, 598. [CrossRef]

18. James, S.W.; Dockney, M.L.; Tatam, R.P. Simultaneous independent temperature and strain measurement using in-fibre Bragg grating sensors. Electron. Lett. 1996, 32, 1133-1334. [CrossRef]

19. Guan, B.O.; Tam, H.Y.; Tao, X.M.; Dong, X.Y. Simultaneous strain and temperature measurement using a superstructure fiber Bragg grating. IEEE Photonics Technol. Lett. 2000, 12, 675-677. [CrossRef]

(C) 2018 by the authors. Licensee MDPI, Basel, Switzerland. This article is an open access article distributed under the terms and conditions of the Creative Commons Attribution (CC BY) license (http://creativecommons.org/licenses/by/4.0/). 